\section{Education and Treatment Management}

\section{REAL-WORLD CLINICAL OUTCOMES AMONG PATIENTS WITH ADVANCED MERKEL CELL CARCINOMA TREATED WITH AVELUMAB IN ACADEMIC MEDICAL CENTERS IN THE UNITED STATES}

\begin{abstract}
${ }^{1}$ Shailender Bhatia, ${ }^{2}$ Paul Nghiem, ${ }^{3} \mathrm{~S}$ Phani Veeranki, ${ }^{4}$ Alejandro Vanegas, ${ }^{5}$ Kristina Lachance, ${ }^{6}$ Lisa Tachiki, ${ }^{3}$ Kevin Chiu, ${ }^{3}$ Emily Boller, ${ }^{7}$ Murtuza Bharmal ${ }^{*}$. ${ }^{1}$ University of Washington, Seattle, WA, USA, Seattle, WA, United States; ${ }^{2}$ UW Medical Center at Lake Union, Seattle, WA, USA, Seattle, WA, United States; ${ }^{3}$ PRECISIONheor, Los Angeles, CA, USA, Los Angeles, CA, United States; ${ }^{4}$ RTI Health Solutions, Parsippany, NJ, USA, Parsippany, NJ, United States; ${ }^{5}$ University of Washington, Seattle, WA, Seattle, WA, United States; ${ }^{6}$ Fred Hutchinson Cancer Research Center, University of Washington, Seattle, WA, USA, Seattle, WA, United States; ${ }^{7} E M D$ Serono, Billerica, MA, USA, Billerica, MA, United States
\end{abstract}

Background Merkel cell carcinoma (MCC) is a rare, aggressive cutaneous neuroendocrine neoplasm with annual incidence rates ranging from 0.13 to 1.6 cases per 100,000 per year. ${ }^{1}$ Chemotherapy for metastatic MCC (mMCC) has high objective response rates (ORRs), but responses are not durable and overall survival (OS) is poor. In March 2017, avelumab (antiPD-L1) was approved for the treatment of mMCC and has demonstrated meaningful survival benefit and durable response. $^{2}$ This study sought to investigate real-world clinical outcomes of avelumab-treated patients with advanced (stage IIIB/IV) MCC in academic medical centers in the United States (US).

Methods A retrospective chart review study of patients with advanced MCC who initiated avelumab between March 1, 2017, and July 31, 2019 was conducted at 6 US academic medical centers across the 4 US census regions. Eligible patients were followed through December 30, 2020. Descriptive analyses were conducted to assess demographics, clinical characteristics, and outcomes. Kaplan-Meier curves were constructed to illustrate real-world duration of response (rwDOR), real-world progression free survival (rwPFS), OS, and time-totreatment discontinuation.

Results Ninety patients with advanced MCC were treated with avelumab, with a median follow-up of 15.0 months (95\% CI, 13.1-17.8). Median age was 68 years; the majority were male $(58 \%)$ and White $(93 \%)$. During the time of avelumab initiation, 74 patients had stage IV MCC and 16 patients had stage IIIB MCC. Primary tumor was located most commonly on the lower limb (38\%), with metastasis primarily to lymph nodes $(67 \%)$ and lung (52\%); $52 \%$ of patients had visceral metastases. Approximately $42 \%$ and $26 \%$ of patients had an Eastern Cooperative Oncology Group (ECOG) performance status of 2 and 3, respectively. Seventy-three patients (81\%) received avelumab as first-line treatment of advanced MCC, whereas $17(19 \%)$ received avelumab as second-line or later. Median duration of avelumab treatment was 13.5 months (95\% CI, 6.4-30.6); 58\% discontinued by the end of follow-up. Patients with avelumab treatment $(\mathrm{n}=90)$ had a rwORR of $73 \%(95 \%$ CI, 64-83), median rwPFS of 24.4 months (95\% CI, 8.3-not reached [NR]), and median OS of 30.7 months (95\% CI, 11.2-NR). Other clinical outcomes by line of avelumab treatment and stage at avelumab initiation are reported in table 1. Conclusions This real-world study of patients with advanced MCC treated with avelumab demonstrates high response rate with durable responses and prolonged survival. The study findings are consistent with the efficacy results demonstrated in pivotal clinical trials ${ }^{2}$ and other recent observational studies. $^{34}$

Abstract 628 Table 1 Clinical outcomes among avelumab-treated patients with advanced MCC by line-of-treatment and stage at avelumab initiation

\begin{tabular}{|c|c|c|c|c|}
\hline Outcomes & $\begin{array}{l}\text { Avelumab 1L } \\
(\mathrm{n}=73)\end{array}$ & $\begin{array}{l}\begin{array}{l}\text { Avelumab } 2 \mathrm{~L}+ \\
(\mathrm{n}=17)\end{array} \\
\end{array}$ & $\begin{array}{l}\begin{array}{l}\text { Stage IIIB } \\
(n=16)\end{array} \\
\end{array}$ & $\begin{array}{l}\text { Stage IV } \\
(n=74)\end{array}$ \\
\hline $\begin{array}{l}\text { Physician assessed } \\
\text { rwORR }(95 \% \mathrm{Cl}), \%\end{array}$ & $75.3(65.2-85.4)$ & $64.7(39.4-90.0)$ & 100 & $67.6(56.6-78.5)$ \\
\hline $\begin{array}{l}\text { Median rwDOR (95\% } \\
\text { Cl), months }\end{array}$ & NR (NR-NR) & 4.6 (1.1-NR) & NR (NR-NR) & NR (7.0-NR) \\
\hline $\begin{array}{l}\text { Median rwPFS ( } 95 \% \\
\text { CI), months }\end{array}$ & 36.1 (9.3-NR) & 6.4 (4.5-NR) & NR (NR-NR) & 10.3 (4.9-NR) \\
\hline $\begin{array}{l}\text { Median OS }(95 \% \mathrm{Cl}) \text {, } \\
\text { months }\end{array}$ & 41.7 (10.2-NR) & 15.9 (4.3-NR) & NR (NR-NR) & $15.9(8.4-41.7)$ \\
\hline
\end{tabular}

Acknowledgements The authors would like to acknowledge all physicians at the respective sites who participated in the data collection process for the study.

\section{REFERENCES}

1. Müller-Richter UDA, Gesierich A, Kübler AC, Hartmann S, Brands RC. Merkel cell carcinoma of the head and neck: recommendations for diagnostics and treatment. Ann Surg Oncol 2017; 24:3430-3437.

2. D'Angelo SP, Bhatia S, Brohl AS, et al. Avelumab in patients with previously treated metastatic Merkel cell carcinoma: long-term data and biomarker analyses from the single-arm phase 2 JAVELIN Merkel 200 trial. J Immunother Cancer 2020;8:e000674.

3. Cowey CL, Liu FX, Kim R, et al. Real-world clinical outcomes with first-line avelu mab in locally advanced/metastatic Merkel cell carcinoma in the USA: SPEAR-Merkel. Future Oncol 2021;17:2339-2350.

4. Levy S, Aarts MJB, Eskens FALM, et al. Avelumab for advanced Merkel cell carcinoma in the Netherlands: a real-world cohort. J Immunother Cancer 2020;8 e001076.

Ethics Approval The study was approved by New England Institutional Review Board.

http://dx.doi.org/10.1136/jitc-2021-SITC2021.628 